\title{
Heat transfer study of phase change material incorporated into a cavity of a hollow brick during melting
}

\author{
O.Youcef Souci ${ }^{1, *}$, Samir Houat ${ }^{1}$ and Mohammed El Ganaoui ${ }^{2}$ \\ ${ }^{1}$ MNEPM Laboratory, MSMPT Group, Mechanical Engineering Department. Abdelhamid Ibn Badis University of Mostaganem, \\ Algeria \\ ${ }^{2}$ LERMAB Laboratory, University of Lorraine, France.
}

\begin{abstract}
The use of Phase Change Materials (PCMs) in the building sector is an attractive solution contributing to the reduction of energy consumption as well as the improvement of the thermal comfort. To achieve this objective, it is required to study the complex phenomena of phase changes when PCM incorporated in different types of building materials. In this study, PCM is incorporating in a cavity of hollow brick and check the effect of natural convection during melting process by compared between two cases, the first the case when there is only a pure conduction and for the second case is when natural convection is not neglected in the upper part of this cavity. For the numerical model, the finite element method is using with modification in the specific heat of PCM accounting for the increase amount of energy, in the form of latent heat, needed to melt the PCM over its melting temperature range. The result showed that the effect of natural convection is limited and can represented by using un equivalent thermal conductivity.
\end{abstract}

\section{Introduction}

Use Nowadays the building sector is a key area for improving energy efficiency [1]. A promising technology of energy storage is the use of phase change materials (PCM) [2]. In this case, the latent heat offers more attractive advantages than sensible heat storage. This is essentially due to its high-energy storage density and to the isothermal nature of the storage process at the melting temperature of the PCM.

The application of thermal energy storage, and in particular PCM, in buildings has been widely studied [38]. The modelling of heat transfer with phase change solid / liquid is usually base on the equation of heat transfer by conduction and solved by a "mobile boundary" method or by another method called "enthalpy". The unknowns of this problem are the temperatures in the solid and liquid phases and the position of the interface. According to the characteristics of the problem, the solution can calculate by analytical or numerical method [9]. A difficulty is that the natural convection in the liquid state isn't always ignoring. Because natural convection in molten PCMs enhances the heat transfer within these systems, a better understanding of the substances used as PCMs as well as

the physical phenomena that take place during phase transition is needed. To solve this problem, an empirical approach is used to estimating an equivalent conductivity of PCM during change phase process. The objective of the present study, is to analyse and validate a model of heat transfer with phase change by studied the melting of PCM incorporated within a cavity of a hollow brick. In the first, natural convection is included by an empirical expression and it will be compared with a second case where heat transfer is only by conduction.

\section{Physical problem}

In this study, a commercial PCM (RT21) is failed in the cavity of hollow brick. The thermos physical properties used of this PCM are shown in Table 1 [10].

Table 1. The thermos physical properties of PCM

\begin{tabular}{|c|c|c|c|c|c|}
\hline Material & $\begin{array}{c}\mathbf{\rho} \\
\mathbf{k g} / \mathbf{m}^{\mathbf{3}}\end{array}$ & $\begin{array}{c}\boldsymbol{\lambda} \\
\mathbf{W} / \mathbf{m} . \mathbf{K}\end{array}$ & $\begin{array}{c}\mathbf{C}_{\mathbf{p}} \\
\mathbf{J} / \mathbf{k g} . \mathbf{K}\end{array}$ & $\begin{array}{c}\mathbf{T}_{\mathbf{f}} \\
{ }^{\circ} \mathbf{C}\end{array}$ & $\begin{array}{c}\mathbf{L}_{\mathbf{f}} \\
\mathbf{k J} / \mathbf{k g}\end{array}$ \\
\cline { 1 - 3 } PCM Solid & 840 & 0.2 & 1400 & & \\
\cline { 1 - 3 } PCM Liquid & 760 & 0.18 & 1700 & 294 & 134 \\
\hline
\end{tabular}

Where $L_{f}, \mathrm{Cp}, \lambda$ are respectively latent heat of fusion, specific heat capacity and thermal conductivity. $T_{f}$ is temperature of fusion.

The geometry used for this study is a simple 2D square enclosure as shown in Fig. 1.

The following assumptions are makes:

- The thermos physical properties of the PCM are independent of temperature but are different for the liquid phases, solid and transition (calculated by linear interpolation between solid and liquid).

- The composition of PCM is considers homogeneous.

- The contact resistances at the interfaces are neglected in the present study.

\footnotetext{
Corresponding author: souci_youcef@yahoo.fr
} 


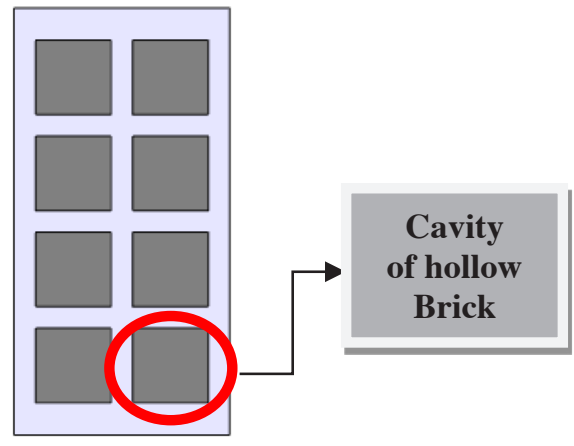

Fig.1. Cavity of hollow Brick.

\section{Governing Equations}

Based on the above assumptions, the equation of conservation of energy in the PCM is writing as follows:

$$
\rho C_{p} \frac{\partial T}{\partial t}+\varepsilon \rho L_{f} \frac{\partial f_{l}}{\partial t}=\lambda \frac{\partial^{2} T}{\partial n^{2}}
$$

With: $\varepsilon=0$ : case of PCM (in liquid or solid phase). $\varepsilon=1$ : $\mathrm{PCM}$ in melting at $\mathrm{T}=\mathrm{T}_{\mathrm{f}}$.

When the natural convection is not negligible, the thermal conductivity is replaced in the equation (1) by an equivalent thermal conductivity $\lambda_{e q}$.

For boundary condition, the left face of the cavity is maintained at a higher temperature than the melting point of the material. For $\mathrm{t}=0, \mathrm{Ti}=294^{\circ} \mathrm{K}$ and varied with a linear increase (up shift $=1^{\circ} \mathrm{C} / \mathrm{h}$ ). The right face is maintained at a lower temperature than the melting point of the material $\left(\mathrm{T}=290^{\circ} \mathrm{K}\right)$. The top and bottom faces are insulated.

\section{Numerical Model}

Solving the solid-liquid interface is one of the major problems in heat transfer calculations. From the literature, there are two types of numerical methods usually used to solve the phase change problem; the enthalpy method and the effective heat capacity method. In this study, the phase change problem is solved using the effective heat capacity method. The effective heat capacity of the PCM during phase change is giving by equation (2) $[11,12]$. The discontinuity of heat capacity is applying in the numerical model with using a logic function (piecewise). Fig. 2 shows the plot of modified heat capacity used in the simulation of PCM.

$$
C_{p}= \begin{cases}C_{\mathrm{p}_{-} \mathrm{s}} & T<T_{S} \\ \frac{C_{\mathrm{p}_{\_} \mathrm{s}}+C_{\mathrm{p}_{-} \mathrm{l}}}{2}+\frac{L}{T_{l}-T_{S}} & T_{s} \leq T \leq T_{l} \\ \mathrm{C}_{\mathrm{p}_{\_} \mathrm{l}} & \mathrm{T}>T_{l}\end{cases}
$$

A solid PCM has then defined as a liquid having an extremely large viscosity, while the liquid PCM possess its true value $\mu_{l}$ when the PCM melt. See the paper $[13,14]$ for more detail. Fig. 3 presents the PCM viscosity as a function of temperature. After several tests, the mesh of 2D construction model consists of

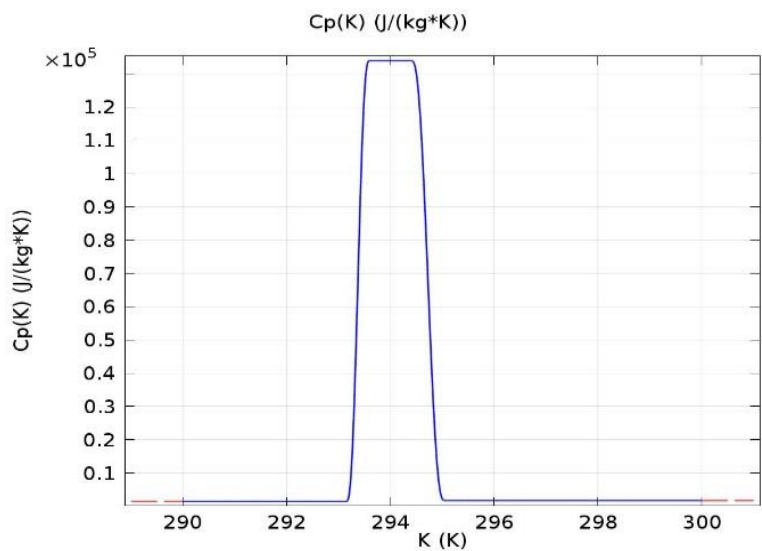

Fig.2. Modified specific heat.

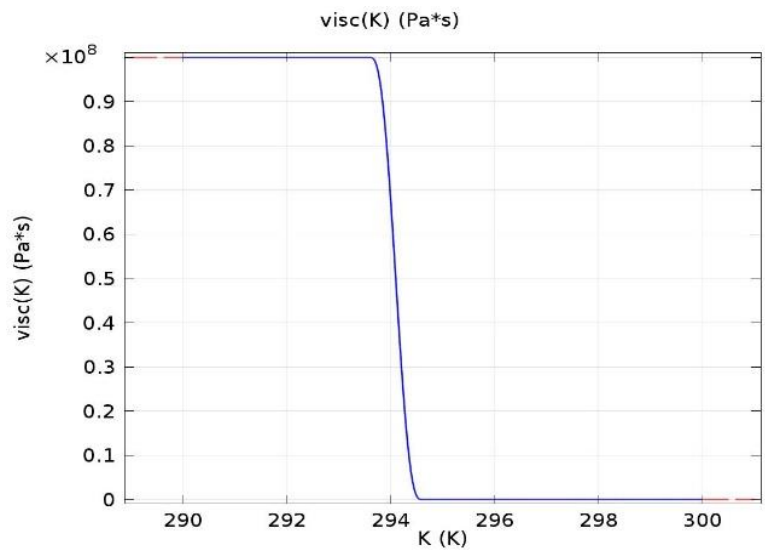

Fig.3. Modified dynamic viscosity.

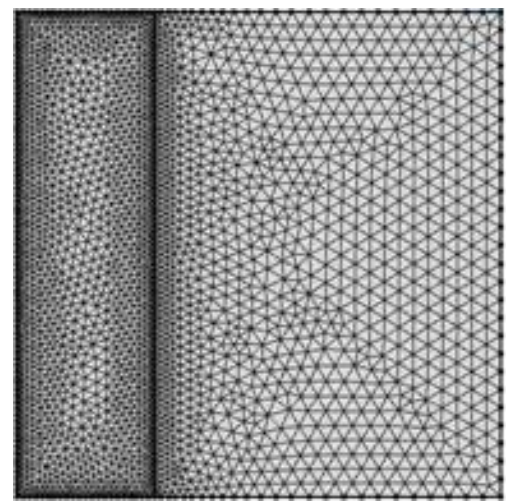

Fig.4. Schematics free triangular and quadrilateral used in this study.

5228 elements. Fig. 4 shows the mesh geometry of this model.

The resolution of the analytical equation (1) gives the transient temperature distribution in the liquid $\mathrm{T}(x, \mathrm{t})$ by [15]:

$$
\begin{array}{ll}
\frac{T_{l}(x, t)-T_{w}}{T_{m}-T_{w}}=\frac{\operatorname{erf}\left(\frac{x}{2 \sqrt{\alpha_{l} t}}\right)}{\operatorname{erf}(k)} \quad 0<x<S \\
\frac{T_{s}(x, t)-T_{o}}{T_{m}-T_{o}}=\frac{\operatorname{erf}\left(\frac{x}{2 \sqrt{\alpha_{s} t}}\right)}{\operatorname{erf}(\gamma \cdot k)} \quad s<x<\infty
\end{array}
$$


With:

$$
\gamma=\sqrt{\frac{\alpha_{l}}{\alpha_{s}}}
$$

Erf and erfc mean respectively the error function and its complement.

Where $\mathrm{S}$ is the position of the interface. $\mathrm{T}_{\mathrm{w}}, \mathrm{T}_{0}, \mathrm{~T}_{\mathrm{m}}$ are respectively the temperature in the left face, in the right face and of melting.

In addition, the parameter $\mathrm{k}$ is giving by the following transcendental equation:

$$
\frac{S t_{l}}{e^{k^{2}} \cdot \operatorname{erf}(k)}-\frac{S t_{s}}{\gamma e^{k^{2} \cdot b^{2}} \cdot \operatorname{erfc}(b \cdot k)}=0
$$

Where $S t_{l}$ and $S t_{s}$ are the values of number of Stefan:

$$
\begin{aligned}
& S t_{l}=\frac{\rho \cdot C_{l} \cdot\left(T_{W}-T_{m}\right)}{L} \\
& S t_{s}=\frac{\rho \cdot C_{s} \cdot\left(T_{m}-T_{0}\right)}{L}
\end{aligned}
$$

The position of the interface $\mathrm{S}$ is giving by the following equation:

$$
S(t)=2 \cdot k \cdot \sqrt{\alpha_{l} \cdot t}
$$

\section{Results and Discussion}

The analytical and numerical results of the interface position during melting for both cases (with and without natural convection) are presented in Fig. 5. In the first case, it has been neglect the natural convection in the liquid zone and the calculation is developed by real thermal conductivity. on the second case, the natural convection has taken into account using an equivalent thermal conductivity. The result showed that the numerical solution obtained is in excellent agreement with the analytical solution. The melting process begins at time $\mathrm{t}=0$, when the left side temperature of the cavity is raised to a constant temperature above the fusion temperature of the PCM. In Fig.6, the melting position interface is shown at different times $(\mathrm{t}=0.25 \mathrm{hrs}, 1 \mathrm{hrs}$ and $1.25 \mathrm{hrs}$ ). Once again, this figure shows the interface position when natural convection is neglected. It has been seen that the convection heat transfer accelerates the process of PCM melting. At the beginning, the thermal transfer has governed by conduction in both cases (with and without natural convection), then, it is the convection, which dominates the thermal transfer into $2 \mathrm{D}$ problems. In addition, it observed that convection dominates in the top of the cavity, shrinking the progression of the solid-liquid interface at the bottom where natural convection has a lesser effect.

The fluctuations of the average temperature in the cold wall of the cavity in the cases studied are shown in figure 7 . The average temperature is changing in the same way for both cases during the first minute by increases linearly until it reaches $294 \mathrm{~K}$ due to the both

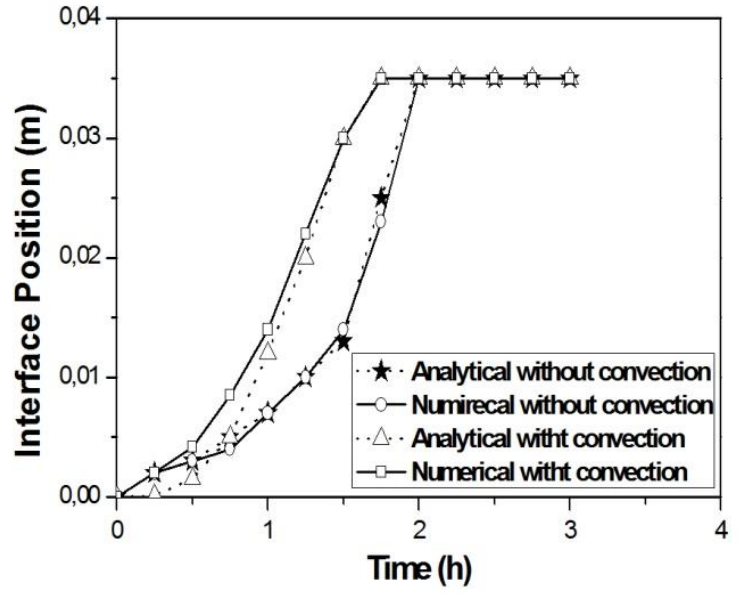

Fig. 5. Melting interface as a function of time.
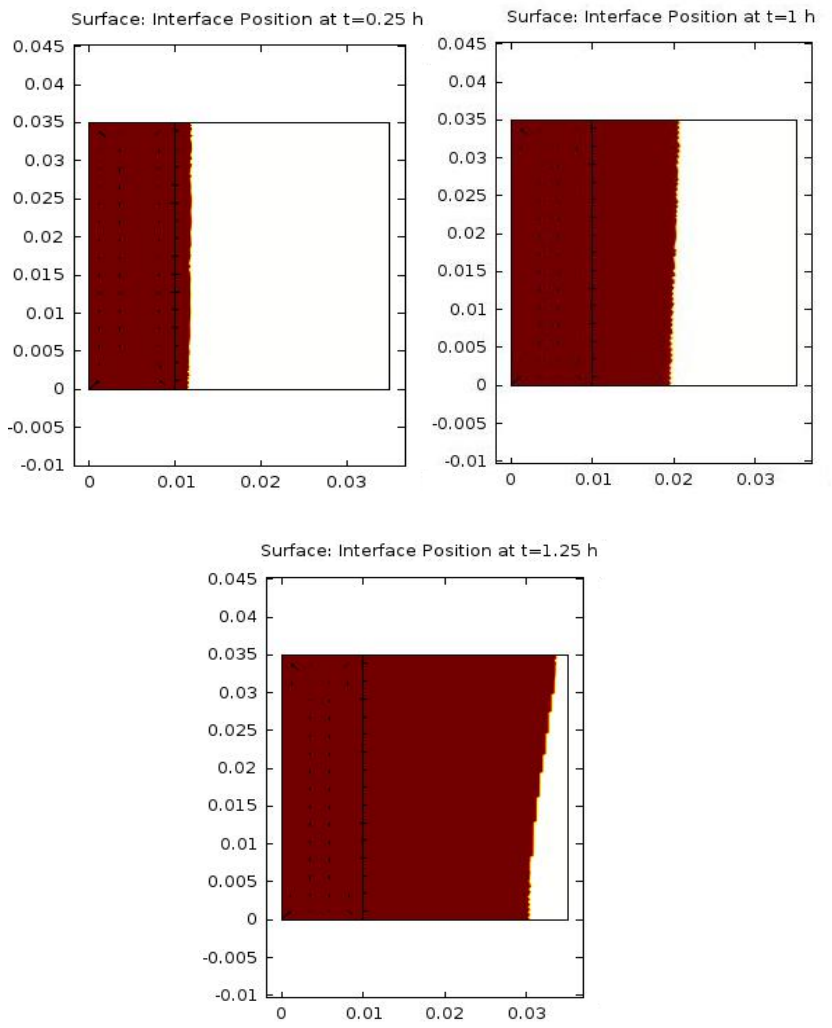

Fig.6. Melting process at several time instants (hr) On the left side: liquid (brown) and solid (white) phase.

sensible heat storage. During this phase, the temperature is changing slightly around $294 \mathrm{~K}$ and this is due to the lack of purity of the used PCM at $100 \%$.

Finally, when the PCM is at liquid state the temperature increases again rapidly by storage of sensible heat. However, there is a clear difference between these two cases. When natural convection has taken into account, it has been observed that the temperature during melting decreases, this phenomenon related to intensification of exchanges in the liquid phase and accelerated of phase change process. At the end of phase change, it has been seen also a second feature represented by an inversion of the temperature gradient in the PCM. This is explaining by migration of 
solids at the bottom of the cavity, related to higher density of solid phase.

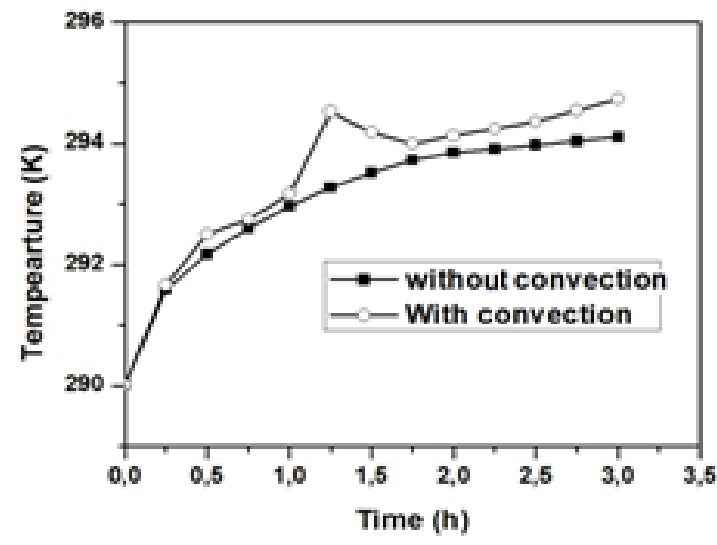

Fig.7. Evaluation of the average temperature of PCM as a function of time.

\section{Conclusion}

In this work, it has been found that the effect of natural convection is limited and can represented by an empirical formula of equivalent thermal conductivity. This approach offers the possibility to having a good precision on the finales results compared to the case of a pure conduction. The study showing also that the physical processes encountered during melting of PCM coupled with convection natural can modelled numerically using the finite element method. The results carried out by simulations are successfully comparing and well fit with analytical results. In addition, natural convection reduced the time needed for storing the latent heat during the melting process. Future work will be about changing the container that includes PCM and check the influence of different surfaces on the process of phase change and we will try other types of PCM with different boundary conditions.

\section{References}

1. N. Jollands, P. Waide, M. Ellis, T Onoda, J Laustsen, K. Tanaka, P. T'Serclaes, I. Barnsley, R. Bradley, A. Meier, Energy Policy 38 (2010).

2. H. Mehling, L.F Cabeza, Heat and Cold Storage with PCM (Springer, Berlin 2002).

3. D. Zhou, C.Y Zhao, Y. Tian, Appl. Energy 92 (2012).

4. A. Abhat. Solar Energy 30 (1983).

5. B. Zalba, J.M Marín, L.F. Cabeza, H. Mehling, Appl. Therm. Eng. 23 (2003).

6. M.M. Farid, A.M. Khudhair, S.A.K. Razack, S. A. Al-Hallaj, Energy Manage 45 (2004).

7. M Kenisarin, K Mahkamov. Renew Sust Energy Rev, 11 (2007).

8. Sharma Atual, VV Tyagi, CR Chen, D Buddhi, Renew Sust Energy Rev, 13,3 (2009).

9. D. Gobin, A. Bricard, Techniques de l'ingénieur BE, Vol.BE2, 32 (2007).
10. Anon, RUBITHERM data sheets for RT21, RT27, RT31 and RT60, RUBITHERM GmbH, Schumann company. (2011).

11. D. Zhang, S A Fung and O Siddiqui., $2^{\text {nd }}$ Canadian Solar Buildings Conference Calgary, (2007).

12. D. Groulx, W. Ogoh, COMSOL Conference 2009, Boston, USA (2009)

13. C. Liu., D. Groulx, COMSOL Conference 2011, Boston, USA (2011)

14. R.E. Murray, D. Groulx, Proceedings of the COMSOL Conference Boston 2011, Boston, USA (2011)

15. A. Alexiades., A.D. Solomon., Hemisphere Pub. Corp., Washington, (1993). 